\title{
On Analysis of Topological Properties for Terbium IV Oxide via Enthalpy and Entropy Measurements
}

\author{
Muhammad Kamran Siddiqui $\mathbb{D}^{\mathrm{D}},{ }^{1}$ Sana Javed, ${ }^{1}$ Lubna Sherin, ${ }^{2}$ Sadia Khalid, ${ }^{1}$ \\ Muhammad Mathar Bashir, ${ }^{2}$ Amir Hassan, ${ }^{1}$ and Mlamuli Dhlamini $\mathbb{D}^{3}$ \\ ${ }^{1}$ Department of Mathematics, COMSATS University Islamabad, Lahore Campus, Lahore 54000, Pakistan \\ ${ }^{2}$ Department of Chemistry, COMSATS University Islamabad, Lahore Campus, Lahore 54000, Pakistan \\ ${ }^{3}$ Department of Applied Mathematics, National University of Science and Technology, Islamabad, Zimbabwe
}

Correspondence should be addressed to Mlamuli Dhlamini; mlamuli.dhlamini@nust.ac.zw

Received 9 June 2021; Accepted 14 August 2021; Published 24 August 2021

Academic Editor: Teodorico C. Ramalho

Copyright ( $) 2021$ Muhammad Kamran Siddiqui et al. This is an open access article distributed under the Creative Commons Attribution License, which permits unrestricted use, distribution, and reproduction in any medium, provided the original work is properly cited.

\begin{abstract}
A relation between topological indices and thermodynamics properties of terbium IV oxide has been established by using a rational method as it was found the most efficient method based on mean squared error (MSE). Terbium IV oxide has huge application as an insulator in modern technologies such as microelectronics, gas detectors, and luminiferous owing to mechanical and thermal stability, high dielectric constant, radiation resistance, and variable electrical conductivity. The chemical graph and topological indices have attracted the research community due to their potential application in discrete mathematics, biology, and chemistry. Our commitment is to investigate topological indices and thermodynamic properties of terbium IV oxide that depend on an innovative data utilitarian. Moreover, a relationship between topological indices and curve fitting has been established as an application point of view. All curve fittings have been found using MATLAB software.
\end{abstract}

\section{Introduction}

Terbium, a rare earth metal, is a member of the $4 \mathrm{f}$ series of the periodic table called lanthanides and has electronic configuration $[\mathrm{Xe}] 4 f^{9} 6 s^{2}$. It is found between the $(n-1) d$ and ns block elements and has properties identical to $\mathrm{d}$-block elements. Due to unfilled $\mathrm{f}$ orbital, electrons are added to the $(n-2)$ level's ' $f$ ' suborbitals. It is silvery white soft metal with a silvery appearance whose melting point ranges from 1000 to 1200 degrees Celsius and is an excellent heat and electricity conductor. Except for promethium, lanthanides are nonradioactive in nature $[1,2]$. The synthesis of terbium IV oxide conventionally uses the precipitation approach, but newly designed self-propagation high-temperature synthesis (SHS) provides the high yield of weakly agglomerated nanosized powder of terbium IV oxide. The flow sheet of this process is given in Figures 1 and 2 [3].

Terbium IV oxide films have huge application as an insulator in microelectronics, gas detectors, and luminophores due to unique properties such as radiation resistance and very small leakage current density besides variable electrical conductivity in different gaseous fluids $[4,5]$. It is found that various oxidation states cause changes in the stoichiometry of terbium IV oxide that predetermines variations in its optical properties, thus making it useful for the aforementioned applications as well as in Fresnel lenses, pigments, antireflection layers, and photoelastic films [6, 7]. Terbium compounds are brightly fluorescent, and most terbium supplies are used to produce green phosphorous worldwide that allow trichromatic lighting [8]. It is also often used as a dopant for fuel-cell materials and crystalline solid-state devices. The large surface basicity, rapid oxygen ion mobility, and interesting catalytic characteristics of earth oxides are well known. These are the qualities which make them a good chemical sensor [9].

The bulk structure of $\mathrm{TbO}_{2}$ is crystallized as a $\mathrm{Tb}_{4} \mathrm{O}_{8}$. In a crystal of terbium oxide, oxygen atoms are located in the cubic closed packed terbium atoms (Figure 1) [10]. 


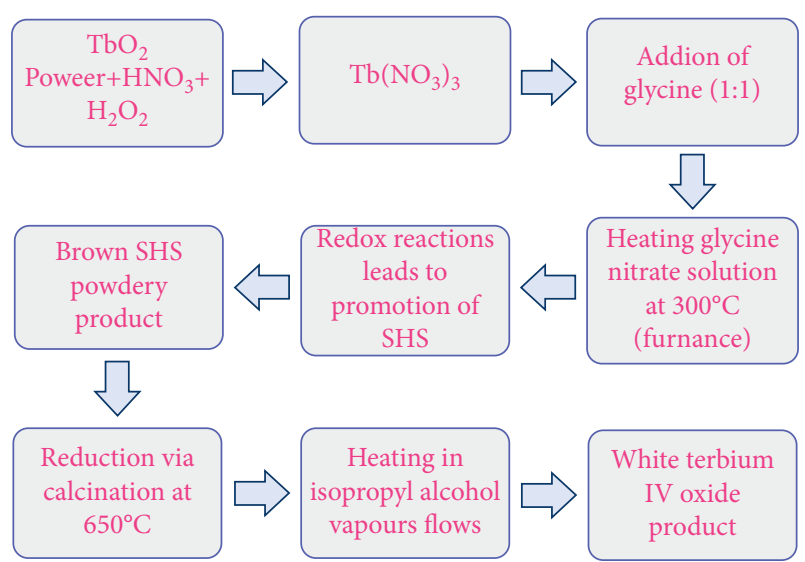

FIGURE 1: Schematic illustration of terbium IV oxide synthesis of by SHS.

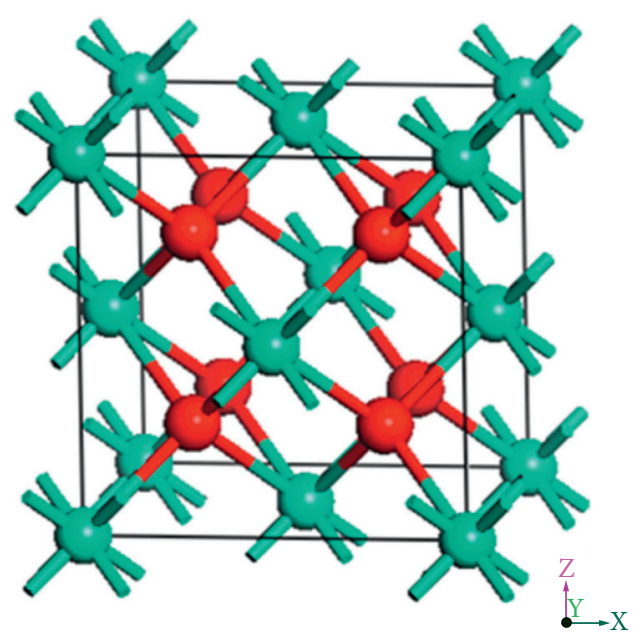

Figure 2: Unit cell structure of terbium oxide [4].

Metal oxide redox reactions are currently regarded being one of the most favorable long-term methods for producing renewable $\mathrm{H}_{2}$ for immediate use in fuel cells. Terbium IV oxide is a promising candidate for thermochemical production of hydrogen through solar thermochemical water splitting (Tb-WS) cycle. The first step of the cycle includes the thermal reduction of $\mathrm{TbO}_{2}$ into $\mathrm{Tb}$ and $\mathrm{O}_{2}$, while the second step involves oxidation of $\mathrm{Tb}$ through a water splitting reaction to produce $\mathrm{H}_{2}$. The unit cell structure of terbium oxide is depicted in Figure 2.

Step 1. Step 1:

$$
\mathrm{TbO}_{2} \longrightarrow \mathrm{Tb}+\mathrm{O}_{2}
$$

Equation (1) is the endothermic reduction.

Step 2.

$$
\mathrm{Tb}+\mathrm{HO}_{2} \longrightarrow \mathrm{TbO}_{2}+2 \mathrm{H}_{2} .
$$

Equation (2) is the endothermic reduction.

\section{Degree-Based Topological Indices}

Let $G=(V, E)$ be a graph where $V$ is the vertex set and $E$ is the edge set of $G$. The degree $\widetilde{I}(a)$ of a vertex $a$ is the number of edges of $G$ incident with $a$.

In 2013, Shirdel et al. [11] introduced the "Hyper-Zagreb index":

$$
\mathrm{HM}=\mathrm{HM}(G)=\sum_{a b \in E(G)}[\widetilde{I}(a)+\widetilde{I}(b)]^{2} .
$$

In 2012, Ghorbani and Azimi [12] defined multiple Zagreb indices as

$$
\begin{aligned}
\operatorname{PM}_{1}(G) & =\prod_{a b \in E(G)}[\widetilde{I}(a)+\widetilde{I}(b)], \\
\operatorname{PM}_{2}(G) & \\
& =\prod_{a b \in E(G)}[\widetilde{I}(a)+\widetilde{I}(b)] .
\end{aligned}
$$

For more details about these indices, see $[13,14]$.

In 1972, Furtula and Gutman $[15,16]$ presented the forgotten topological index which was characterized as

$$
F(G)=\sum_{a b \in E(G)}\left(\widetilde{I}(a)^{2}+\widetilde{I}(b)^{2}\right) .
$$

Furtula et al. [17] introduced the "augmented Zagreb index":

$$
\operatorname{AZI}(G)=\sum_{a b \in E(G)}\left(\frac{\widetilde{I}(a) \times \widetilde{I}(b)}{\widetilde{I}(a)+\widetilde{I}(b)-2}\right)^{3} .
$$

The Balaban index $[18,19]$ is a topological index based on order $n$ and size $m$ of graph $G$ :

$$
J(G)=\frac{m}{m-n+2} \sum_{a b \in E(G)} \frac{1}{\sqrt{I(a) \times \widetilde{I}(b)}},
$$

where $\widetilde{I}(a), \widetilde{I}(b)$ are the degrees of the vertices $a, b \in V(G)$.

For more details about these indices, see [20-22].

The redefined version of the Zagreb indices was defined by Ranjini et al. [23].

$$
\begin{aligned}
& \operatorname{ReZG}_{1}(G)=\sum_{a b \in E(G)} \frac{\widetilde{I}(a)+\widetilde{I}(b)}{\widetilde{I}(a) \times \widetilde{I}(b)}, \\
& \operatorname{ReZG}_{2}(G)=\sum_{a b \in E(G)} \frac{\widetilde{I}(a) \times \widetilde{I}(b)+\widetilde{I}(b)}{\widetilde{I}(a)} \\
& \operatorname{ReZG}_{3}(G)=\sum_{a b \in E(G)} \widetilde{I}(a) \times \widetilde{I}(b)(\widetilde{I}(a)+\widetilde{I}(b)) .
\end{aligned}
$$

For more details about these indices, see [24-31].

\section{Results for Terbium Oxide $\left(\mathrm{TbO}_{2}\right)$}

The number of vertices and edges of the structure of terbium oxide denoted by $\left(\mathrm{TbO}_{2}\right)$ are $22 \mathrm{mn}$ and $32 \mathrm{mn}$, respectively. There are three type of vertices in $\mathrm{TbO}_{2}$, namely, the vertices of degree 1,2 , and 4 , respectively. The vertex partition of the 
vertex set $\mathrm{TbO}_{2}$ is presented in Table 1 . Also, the edge partition of $\mathrm{TbO}_{2}$ based on degrees of end vertices of each edge is depicted in Table 2.

(i) The Hyper-Zagreb index:
The hyper Zagreb index is computed by using Table 2 as follows:

$$
\begin{aligned}
\operatorname{HM}(G)= & \sum_{a b \in E(G)}(\widetilde{I}(a)+\widetilde{I}(b))^{2}, \\
= & \sum_{a b \in E_{1}(G)}(\widetilde{I}(a)+\widetilde{I}(b))^{2}+\sum_{a b \in E_{2}(G)}(\widetilde{I}(a)+\widetilde{I}(b))^{2}+\sum_{a b \in E_{3}(G)}(\widetilde{I}(a)+\widetilde{I}(b))^{2}, \\
= & \operatorname{HM}(G)=(12 m n-2 n-2 m)(1+4)^{2}+(4 m n-2 m-2 n)(2+4)^{2} \\
& +(16 m n+4 m+4 n)(4+4)^{2}, \\
= & \operatorname{HM}(G)=4540 m n+902 m+902 n .
\end{aligned}
$$

(ii) The first and second multiplicative Zagreb index:

The first multiplicative Zagreb index is computed as

$$
\begin{aligned}
& \mathrm{PM}_{1}(G)=(1+4)^{(12 m n-2 n-2 m)} \cdot(2+4)^{(4 m n-2 m-2 n)} \cdot(4+4)^{(16 m n+4 m+4 n)}, \\
& \mathrm{PM}_{1}(G)=5^{(12 m n-2 n-2 m)} \cdot 6^{(4 m n-2 m-2 n)} \cdot 8^{(16 m n+4 m+4 n)}, \\
& \operatorname{PM}_{1}(G)=\prod_{a b \in E(G)}((\widetilde{I} a)+\widetilde{I}(b))=\prod_{a b \in E_{1}(G)}(\widetilde{I}(a)+\widetilde{I}(b))+\prod_{a b \in E_{2}(G)}(\widetilde{I}(a)+\widetilde{I}(b))+\prod_{a b \in E_{3}(G)}(\widetilde{I}((a)+\widetilde{I}((b)) .
\end{aligned}
$$

The second multiplicative Zagreb index is computed as

$$
\begin{aligned}
& \mathrm{PM}_{2}(G)=\prod_{a b \in E(G)}((\widetilde{I} a)+\widetilde{I}(b))=\prod_{a b \in E_{1}(G)}(\widetilde{I}(a)+\widetilde{I}(b))+\prod_{a b \in E_{2}(G)}(\widetilde{I}(a)+\widetilde{I}(b))+\prod_{a b \in E_{3}(G)}(\widetilde{I}((a)+\widetilde{I}((b)), \\
& \mathrm{PM}_{2}(G)=(1+4)^{(12 m n-2 n-2 m)} \cdot(2+4)^{(4 m n-2 m-2 n)} \cdot(4+4)^{(16 m n+4 m+4 n)}, \\
& \mathrm{PM}_{2}(G)=4^{(12 m n-2 n-2 m)} \cdot 8^{(4 m n-2 m-2 n)} \cdot 16^{(16 m n+4 m+4 n)} .
\end{aligned}
$$

(iii) The first and second multiplicative Zagreb index: The numerical representation of the above computed results is presented in Table 3 . (iv) The forgotten index:

The forgotten index is computed as

$$
\begin{aligned}
F(G)= & \operatorname{sum}_{a b \in E(G)}\left(\widetilde{I}(a)^{2}+\widetilde{I}(b)^{2}\right), \\
= & F(G)=\sum_{a b \in E_{1}}\left[\widetilde{I}(a)^{2}+\widetilde{I}(b)^{2}\right]+\sum_{a b \in E_{2}}\left[\widetilde{I}(a)^{2}+\widetilde{I}(b)^{2}\right]+\sum_{a b \in E_{3}}\left[\widetilde{I}(a)^{2}+\widetilde{I}(b)^{2}\right], \\
= & (12 m n-2 n-2 m)\left((1)^{2}+(4)^{2}\right)+(4 m n-2 m-2 n)\left((2)^{2}+(4)^{2}\right)+(16 m n+4 m+4 n)\left((4)^{2}+(4)^{2}\right), \\
= & (12 m n-2 n-2 m)(17)+(4 m n-2 m-2 n)(20)+(16 m n+4 m+4 n)(32) \\
& \cdot 796 m n+54 m+54 n .
\end{aligned}
$$


TABLE 1: Vertex partition of $\mathrm{TbO}_{2}$ based on the degree of vertex.

\begin{tabular}{lcc}
\hline$\widetilde{I}(a)$ & Frequency & Set of vertices \\
\hline 1 & $4 m n+4$ & $V_{1}$ \\
2 & $2 n+2 m-4$ & $V_{2}$ \\
4 & $18 m n-2 m-2 n$ & $V_{3}$ \\
\hline
\end{tabular}

TABLE 2: Edge partition of $\mathrm{TbO}_{2}$.

\begin{tabular}{lcc}
\hline$(\widetilde{I}(a), \widetilde{I}(b))$ & Frequency & Set of edges \\
\hline$(1,4)$ & $12 m n-2 n-2 m$ & $E_{1}$ \\
$(2,4)$ & $4 m n-2 m-2 n$ & $E_{2}$ \\
$(4,4)$ & $16 m n+4 m+4 n$ & $E_{3}$ \\
\hline
\end{tabular}

TABle 3: Comparison of indices $\mathrm{HM}(G), \mathrm{PM}_{2}, \mathrm{PM}_{2}(G)$.

\begin{tabular}{lccc}
\hline$[m, n]$ & $\mathrm{HM}(G)$ & $\mathrm{PM}_{1}(G)$ & $\mathrm{PM}_{2}(G)$ \\
\hline$[1,1]$ & 6344 & $1.844 \times 10^{27}$ & $5.192296859 \times 10^{33}$ \\
{$[2,2]$} & 21768 & $2.699 \times 10^{106}$ & $4.332296397 \times 10^{127}$ \\
{$[3,3]$} & 46272 & $3.132 \times 10^{237}$ & $5.808659799 \times 10^{281}$ \\
{$[4,4]$} & 79856 & $2.883 \times 10^{420}$ & $1.251505764 \times 10^{496}$ \\
{$[5,5]$} & 122520 & $2.105 \times 10^{655}$ & $4.333002103 \times 10^{770}$ \\
{$[6,6]$} & 174264 & $1.219 \times 10^{942}$ & $2.410705053 \times 10^{1105}$ \\
{$[7,7]$} & 235088 & $5.600 \times 10^{1280}$ & $2.155253650 \times 10^{1500}$ \\
\hline
\end{tabular}

The Augmented Zagreb index is computed as follows:

(v) The Augmented Zagreb index:

$$
\begin{aligned}
\operatorname{AZI}(G) & =\sum_{a b \in E(G)}\left(\frac{\widetilde{I}(a) \times \widetilde{I}(b)}{\widetilde{I}(a)+\widetilde{I}(b)-2}\right)^{3}, \\
\operatorname{AZI}(G) & =\sum_{a b \in E_{1}}\left(\frac{\widetilde{I}(a) \times \widetilde{I}(b)}{\widetilde{I}(a)+\widetilde{I}(b)-2}\right)^{3}+\sum_{a b \in E_{2}}\left(\frac{\widetilde{I}(a) \times \widetilde{I}(b)}{\widetilde{I}(a)+\widetilde{I}(b)-2}\right)^{3}+\sum_{a b \in E_{3}}\left(\frac{\widetilde{I}(a) \times \widetilde{I}(b)}{\widetilde{I}(a)+\widetilde{I}(b)-2}\right)^{3}, \\
& =\sum_{a b \in E_{1}}\left(\frac{1 \times 4}{1+4-2}\right)^{3}+\sum_{a b \in E_{2}}\left(\frac{2 \times 4}{2+4-2}\right)^{3}+\sum_{a b \in E_{3}}\left(\frac{4 \times 4}{4+4-2}\right)^{3}, \\
& =\frac{64}{27}(12 m n-2 n-2 m)+8(4 m n-2 m-2 n)+\frac{512}{27}(16 m n+4 m+4 n), \\
& =\frac{9824 m n}{27}+\frac{496 m}{9}+\frac{496 n}{9} .
\end{aligned}
$$

(vi) The Balaban index:

The Balaban index is computed as

$$
\begin{aligned}
J(G) & =\frac{q}{q-p+2} \sum_{a b \in E(G)} \frac{1}{\sqrt{\tilde{I}(a) \times \widetilde{I}(b)}}, \\
J(G) & =\frac{q}{q-p+2}\left[\sum_{a b \in E_{1}} \frac{1}{\sqrt{I(a) \times \widetilde{I}(b)}}+\sum_{a b \in E_{2}} \frac{1}{\sqrt{\tilde{I}(a) \times \widetilde{I}(b)}}+\sum_{a b \in E_{3}} \frac{1}{\sqrt{I(a) \times \widetilde{I}(b)}}\right], \\
& =\frac{q}{q-p+2}\left[\sum_{a b \in E_{1}} \frac{1}{\sqrt{1 \times 4}}+\sum_{a b \in E_{2}} \frac{1}{\sqrt{2 \times 4}}+\sum_{a b \in E_{3}} \frac{1}{\sqrt{4 \times 4}}\right], \\
& =\frac{22 m n}{10 m n-2} \times\left[\frac{1}{2}(12 m n-2 n-2 m)+\frac{1}{2 \sqrt{2}}(4 m n-2 m-2 n)+\frac{1}{4}(16 m n+4 m+4 n)\right] .
\end{aligned}
$$


The numerical representation of the abovecomputed results is presented in Table 4.

(vii) The redefined Zagreb indices:
The redefined Zagreb indices are computed as

$$
\begin{aligned}
& \operatorname{Re}_{1}(G)=\sum_{a b \in E(G)} \frac{\widetilde{I}(a)+\widetilde{I}(b)}{\widetilde{I}(a) \times \widetilde{I}(b)} \\
& \operatorname{ReG}_{1}(G)=\sum_{a b \in E_{1}} \frac{\widetilde{I}(a)+\widetilde{I}(b)}{\widetilde{I}(a) \times \widetilde{I}(b)}+\sum_{a b \in E_{2}} \frac{\widetilde{I}(a)+\widetilde{I}(b)}{\widetilde{I}(a) \times \widetilde{I}(b)}+\sum_{a b \in E_{3}} \frac{\widetilde{I}(a)+\widetilde{I}(b) \times \widetilde{I}(b)}{\widetilde{I}(a)} \\
& =\sum_{a b \in E_{1}} \frac{1+4}{1 \times 4}+\sum_{a b \in E_{2}} \frac{2+4}{2 \times 4}+\sum_{a b \in E_{3}} \frac{4+4}{4 \times 4} \\
& \left.=\frac{5}{4}(12 m n-2 n-2 m)+\frac{3}{4}(4 m n-2 m-2 n)+\frac{1}{2}(16 m n+4 m+4 n)\right), \\
& \operatorname{Re}_{2}(G)=\sum_{a b \in E(G)} \frac{\widetilde{I}(a)+\widetilde{I}(b)}{\widetilde{I}(a) \times \widetilde{I}(b)} \\
& \operatorname{ReG}_{2}(G)=\sum_{a b \in E_{1}} \frac{\widetilde{I}(a)+\widetilde{I}(b)}{\widetilde{I}(a) \times \widetilde{I}(b)}+\sum_{a b \in E_{2}} \frac{\widetilde{I}(a)+\widetilde{I}(b)}{\widetilde{I}(a) \times \widetilde{I}(b)}+\sum_{a b \in E_{3}} \frac{\widetilde{I}(a)+\widetilde{I}(b) \times \widetilde{I}(b)}{\widetilde{I}(a), ~} \\
& =\sum_{a b \in E_{1}} \frac{1 \times 4}{1+4}+\sum_{a b \in E_{2}} \frac{2 \times 4}{2+4}+\sum_{a b \in E_{3}} \frac{4 \times 4}{4+4} \\
& \left.=\frac{4}{5}(12 m n-2 n-2 m)+\frac{4}{3}(4 m n-2 m-2 n)+(2)(16 m n+4 m+4 n)\right) \text {, } \\
& \operatorname{ReG}_{3}(G)=\sum_{a b \in E(G)}[\widetilde{I}(a) \widetilde{I}(b)(\widetilde{I}(a)+\widetilde{I}(b))], \\
& \operatorname{Re}_{3}(G)=\sum_{a b \in E_{1}}[\widetilde{I}(a) \widetilde{I}(b)(\widetilde{I}(a)+\widetilde{I}(b))] \\
& +\sum_{a b \in E_{2}}[\widetilde{I}(a) \widetilde{I}(b)(\widetilde{I}(a)+\widetilde{I}(b))]+\sum_{a b \in E_{3}}[\widetilde{I}(a) \widetilde{I}(b)(\widetilde{I}(a)+\widetilde{I}(b))], \\
& =20(12 m n-2 n-2 m)+48(4 m n-2 m-2 n)+128(16 m n+4 m+4 n), \\
& =2480 m n+376 m+376 n \text {. }
\end{aligned}
$$

The numerical representation of the above computed results is presented in Table 5 .

\section{Heat of Formation and Entropy of Terbium IV Oxide}

The topological indices $\operatorname{HM}(G), \operatorname{PM}(G), \operatorname{PM} 2(G), F(G)$, $\operatorname{AZI}(G), J(G), \operatorname{Re} G 1(G), \operatorname{Re} G 2(G)$, and $\operatorname{Re} G 3(G)$ were calculated for various numbers of unit cells of terbium IV oxide. The thermodynamic properties of terbium IV oxide, such as heat of formation or enthalpy and entropy, are related to these indices $\operatorname{HM}(G), \operatorname{PM}(G), \operatorname{PM} 2(G), F(G)$, $\operatorname{AZI}(G), J(G), \operatorname{Re} G 1(G), \operatorname{Re} G 2(G)$, and $\operatorname{Re} G 3(G)$. Terbium
IV oxide has a standard molar enthalpy of $-972.2 \mathrm{kJmol}^{-1}$, and the standard molar enthalpy for one formula unit was calculated by dividing it by Avogadro's number. The enthalpy of a cell was determined by multiplying the acquired value by the number of formula units within the cell. The enthalpy of terbium IV oxide is directly proportional to its crystal size and increases as the number of unit cells increases, according to these findings. The entropy of terbium IV oxide was calculated using the same process. The molar standard entropy of terbium IV oxide is $86.9 \mathrm{Jmol}^{-1} \mathrm{~K}^{-1}$. The result was then determined by multiplying the number of formula units in a single unit cell. If the number of cells gets exponential, the entropy value decreases. The downward 
TABle 4: Comparison of indices for $F(G), \mathrm{AZI}(G), J(G) \mathrm{TbO}_{2}$.

\begin{tabular}{lccc}
\hline$[m, n]$ & $F(G)$ & AZI $(G)$ & $J(G)$ \\
\hline$[1,1]$ & 904 & 474.07 & 27.5 \\
{$[2,2]$} & 3400 & 1675.85 & 99.18 \\
{$[3,3]$} & 7488 & 3605.33 & 221.59 \\
{$[4,4]$} & 13168 & 6262.52 & 394.26 \\
{$[5,5]$} & 20440 & 9647.41 & 617.16 \\
{$[6,6]$} & 29304 & 13760 & 890.28 \\
{$[7,7]$} & 39760 & 18600.3 & 1213.63 \\
\hline
\end{tabular}

Table 5: Comparison of $\operatorname{Re} G_{1}(G), \operatorname{Re}_{2}(G), \operatorname{Re}_{3}(G)$ indices for $\mathrm{TbO}_{2}$.

\begin{tabular}{lccc}
\hline$[m, n]$ & $\operatorname{Re}_{1}(G)$ & $\operatorname{Re}_{2}(G)$ & $\operatorname{Re}_{3}(G)$ \\
\hline$[1,1]$ & 22 & 54.4 & 3232 \\
{$[2,2]$} & 96 & 202.67 & 11424 \\
{$[3,3]$} & 222 & 444.8 & 24576 \\
{$[4,4]$} & 400 & 780.8 & 42688 \\
{$[5,5]$} & 630 & 1210.67 & 65760 \\
{$[6,6]$} & 912 & 1734.4 & 93792 \\
{$[7,7]$} & 1246 & 2352 & 126784 \\
\hline
\end{tabular}

trend is the complete opposite of the heat of formation. The graphical representation is depicted in Figures 3-5.

The values for entropy and heat of formation of terbium oxide $\left(\mathrm{TbO}_{2}\right)$ for $1 \leq m \leq 7$ and $1 \leq n \leq 7$ corresponding to different formula units are computed in Table 6 .

\section{A Mathematical Description of Heat of Formation and Entropy of Terbium Oxide in Terms of Topological Indices}

Computational approaches integrated with other disciplines of science provide a coherent way to understand a scientific problem more intensely. Usually, it is not apprehending to understand a problem based on just one science discipline, so adding some computational approaches to the study might provide a clear picture which helps to investigate the underlying phenomenon more deeply and clearly. Developing a mathematical model to describe the dynamics of objects or components involved in a study provides a very convenient mode to tackle and analyze the matter of concern. At present, software technology is playing a vital role in conducting such studies, as they provide more efficient programs to convert an experimental study into a mathematical problem and analyze it.

In this section, we have developed mathematical models to represent the thermodynamic properties, namely, heat of formation and entropy, in the form of topological indices of terbium oxide. This might provide us an efficient way to understand the molecular structure of terbium oxide based on its chemical graph structure properties. We have used the software of MATLAB to estimate such models. There are several built-in methods to fit curves between two variables. We tried all of them and found the rational method as the most efficient one as it was providing the least residuals between empirical and fitted values. Tables 7 and 8 contain root mean squared error (RMSE), sum of squared error (SSE), and $R^{2}$, where ratij represents rational fit with numerator degree $i$ and denominator degree $j$.

5.1. General Models for Indices vs. Heat of Formation. In this section, a mathematical framework has been developed between each topological index and heat of formation (HoF) of terbium oxide. All the fitted curves are shown in Figures 6-12, whereas the estimated parametric values are provided in Tables 9-15.

$$
\begin{aligned}
\mathrm{HoF}(\mathrm{HM}) & =\frac{p_{1}}{(\mathrm{HM})^{3}+q_{1} \times(\mathrm{HM})^{2}+q_{2} \times \mathrm{HM}+q_{3}} \\
\mathrm{HoF}(F) & =\frac{p_{1} \times F^{2}+p_{2} \times F+p_{3}}{F^{3}+q_{1} \times F^{2}+q_{2} \times F+q_{3}} . \\
\operatorname{HoF}(\mathrm{AZI}) & =\frac{p_{1} \times(\mathrm{AZI})^{2}+p_{2} \times \mathrm{AZI}+p_{3}}{(\mathrm{AZI})^{3}+q_{1} \times(\mathrm{AZI})^{2}+q_{2} \times \mathrm{AZI}+q_{3}} . \\
\operatorname{HoF}(J) & =\frac{p_{1} \times J^{2}+p_{2} \times J+p_{3}}{J^{3}+q_{1} \times J^{2}+q_{2} \times J+q_{3}} . \\
\operatorname{HoF}\left(\operatorname{Re} G_{1}\right) & =\frac{p_{1} \times \operatorname{Re} G_{1}+p_{2}}{\left(\operatorname{Re} G_{1}\right)^{3}+q_{1} \times\left(\operatorname{Re} G_{1}\right)^{2}+q_{2} \times \operatorname{ReG} G_{1}+q_{3}} .
\end{aligned}
$$




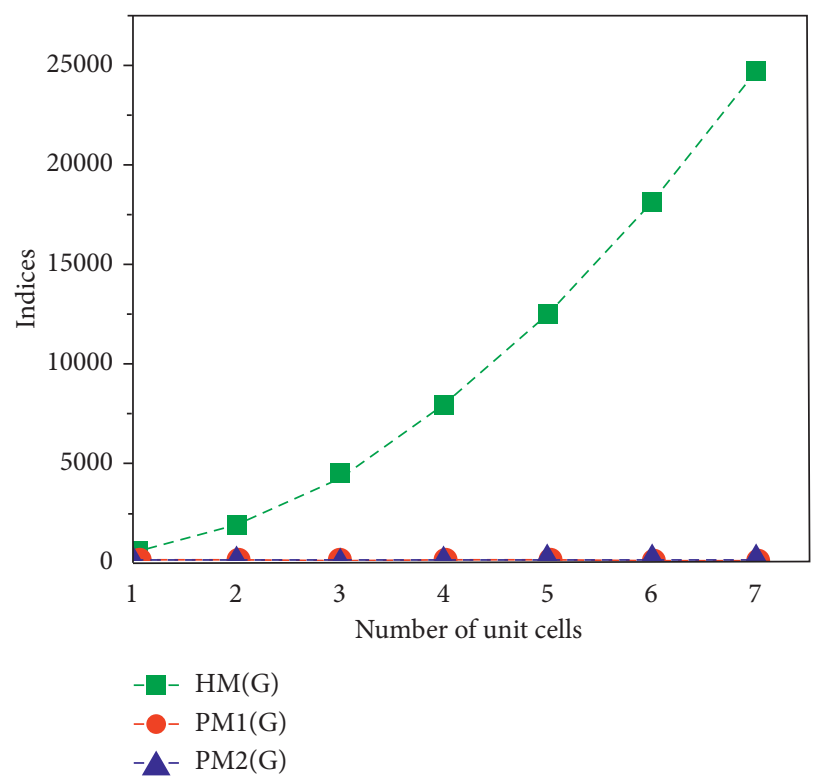

Figure 3: $\operatorname{HM}(G), P M_{1}(G), \mathrm{PM}_{2}(G)$ indices vs. unit cell.

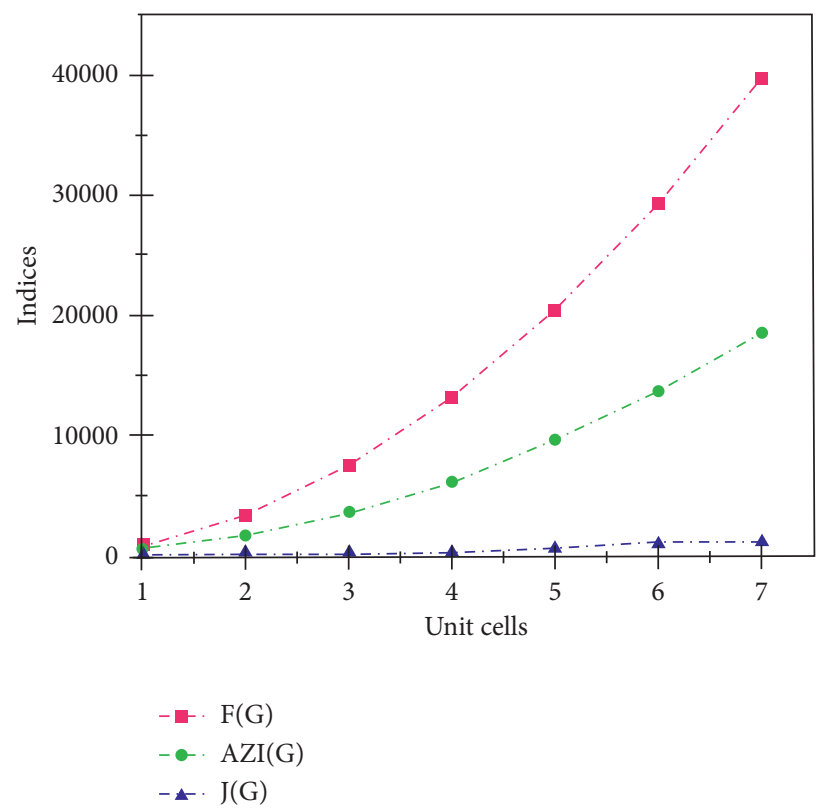

Figure 4: Comparison of indices $\mathrm{F}(\mathrm{G}), \mathrm{AZI}(\mathrm{G})$, and $\mathrm{J}(\mathrm{G})$ for $\mathrm{TbO}_{2}$.

$\operatorname{HoF}\left(\operatorname{ReG}_{2}\right)=\frac{p_{1}}{\left(\operatorname{ReG}_{2}\right)^{3}+q_{1} \times\left(\operatorname{ReG}_{2}\right)^{2}+q_{2} \times \operatorname{ReG}_{2}+q_{3}}$.

$$
\operatorname{HoF}\left(\operatorname{Re}_{3}\right)=\frac{p_{1}}{\left(\operatorname{Re} G_{3}\right)^{3}+q_{1} \times\left(\operatorname{Re} G_{3}\right)^{2}+q_{2} \times \operatorname{Re} G_{3}+q_{3}} .
$$

Coefficients (with 95\% confidence interval (CI)) are given in Table 9.

Coefficients (with 95\% confidence bounds) are given in Table 10.

Coefficients (with $95 \%$ confidence bounds) are given in Table 11.

Coefficients (with $95 \%$ confidence bounds) are given in Table 12.

Coefficients (with $95 \%$ confidence bounds) are given in Table 13. 


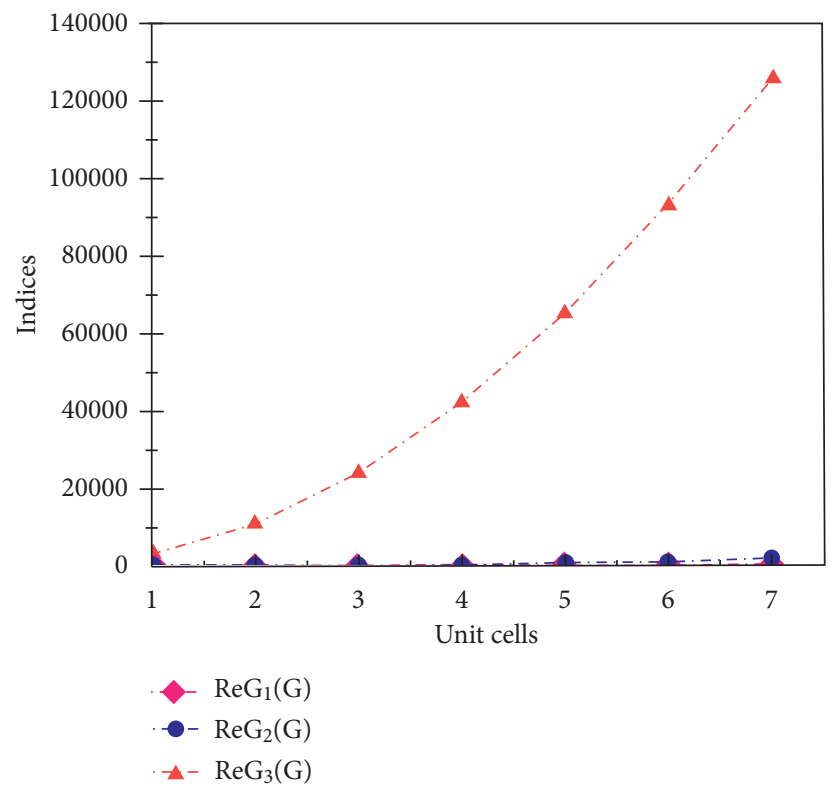

Figure 5: Comparison of $\operatorname{Re}_{1}(G), \operatorname{Re}_{2}(G)$, and $\operatorname{Re}_{3}(G)$ indices for $\mathrm{TbO}_{2}$.

TABLE 6: Values of heat of formation and entropy for different formula units of $\mathrm{TbO}_{2}$.

\begin{tabular}{lccc}
\hline$[m, n]$ & Formula units & Heat of formation $\times 10^{-21} \mathrm{~kJ}$ & Entropy $\times 10^{-22} \mathrm{~kJ}$ \\
\hline$[1,1]$ & 4 & -6.456 & 5.768 \\
{$[2,2]$} & 16 & -0.258 & 0.2307 \\
{$[3,3]$} & 32 & -0.516 & 0.5191 \\
{$[4,4]$} & 64 & -0.0103 & 0.9228 \\
{$[5,5]$} & 100 & -0.0614 & 0.0144 \\
{$[6,6]$} & 144 & -0.0232 & 0.0207 \\
{$[7,7]$} & 196 & -0.0316 & 0.0286 \\
\hline
\end{tabular}

TABLE 7: Goodness of fit for heat of formation vs. indices for $\mathrm{TbO}_{2}$.

\begin{tabular}{lcccc}
\hline Index & Fit type & SSE & $R^{2}$ & RMSE \\
\hline $\mathrm{HM}(G)$ & rat03 & 0.0121 & 0.9996 & 0.0635 \\
$F(G)$ & rat23 & 0.003584 & 0.9999 & 0.05987 \\
$\mathrm{AZI}(G)$ & rat23 & 0.0001696 & 1.000 & 0.01302 \\
$J(G)$ & rat23 & 0.001481 & 1.000 & 0.03849 \\
$\operatorname{Re} G_{1}(G)$ & rat13 & 0.003094 & 0.9999 & 0.03933 \\
$\operatorname{Re} G_{2}(G)$ & rat03 & 0.005014 & 0.9999 & 0.04088 \\
$\operatorname{Re} G_{3}(G)$ & rat03 & 0.01194 & 0.9997 & 0.06308 \\
\hline
\end{tabular}

TABle 8: Goodness of fit for entropy vs. indices for $\mathrm{TbO}_{2}$.

\begin{tabular}{lcccc}
\hline Index & Fit type & SSE & $R^{2}$ & $R M S E$ \\
\hline $\mathrm{HM}(G)$ & rat13 & 0.001071 & 1.000 & 0.02314 \\
$F(G)$ & rat13 & 0.001065 & 1.000 & 0.02307 \\
$\mathrm{AZI}(G)$ & rat03 & 0.001078 & 1.000 & 0.01895 \\
$J(G)$ & rat05 & 0.001031 & 1.000 & 0.0321 \\
$\operatorname{Re} G_{1}(G)$ & rat14 & 0.001118 & 1.000 & 0.03344 \\
$\operatorname{ReG}(G)$ & rat05 & 0.0004906 & 1.000 & 0.02215 \\
$\operatorname{ReG} G_{3}(G)$ & rat13 & 0.001069 & 1.000 & 0.02312 \\
\hline
\end{tabular}




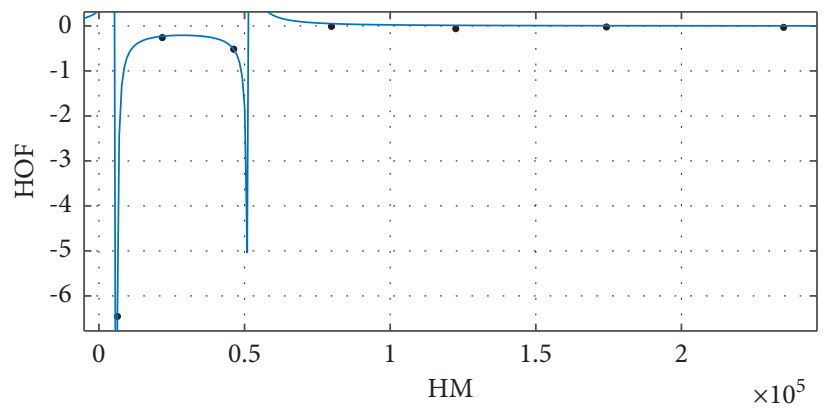

- HOF vs. HM

- HM

Figure 6: $\mathrm{HoF}(\mathrm{HM})$.

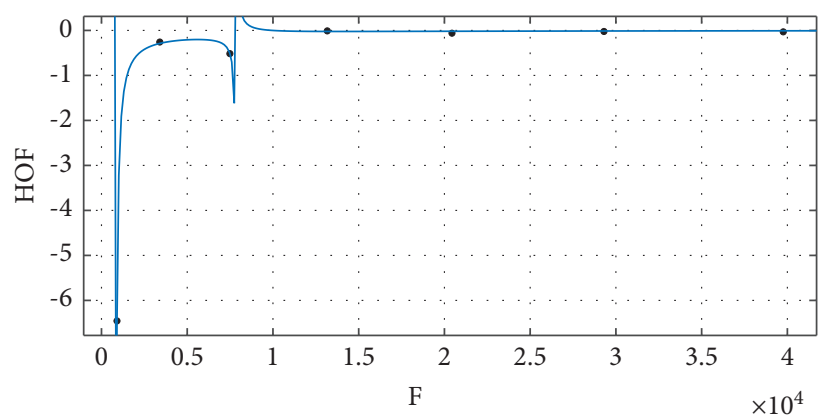

- HOF vs. F

$\mathrm{F}$

Figure 7: $\operatorname{HoF}(F)$.

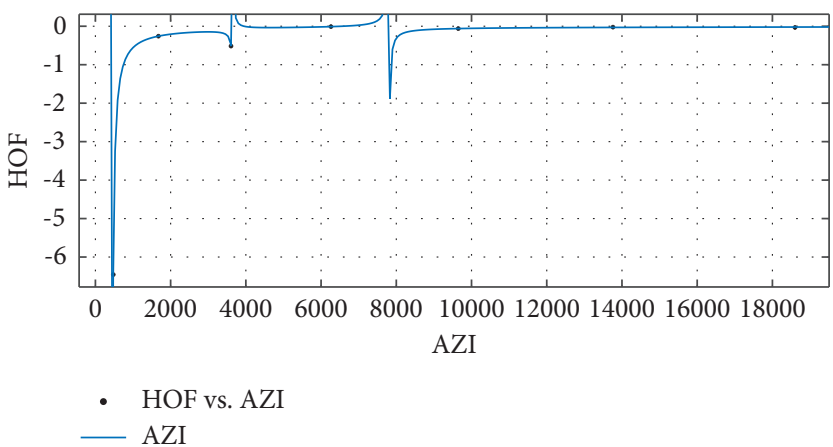

Figure 8: HoF (AZI).

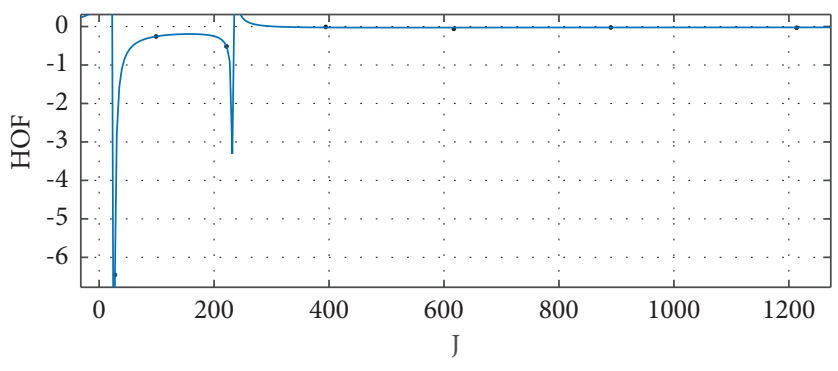

- HOF vs. J

Figure 9: $\operatorname{HoF}(J)$. 


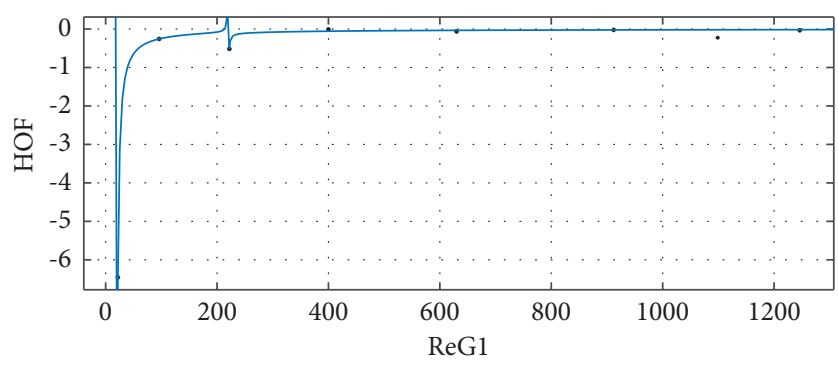

- HOF vs. ReG1

- ReG1

Figure 10: $\operatorname{HoF}\left(\operatorname{ReG}_{1}\right)$.

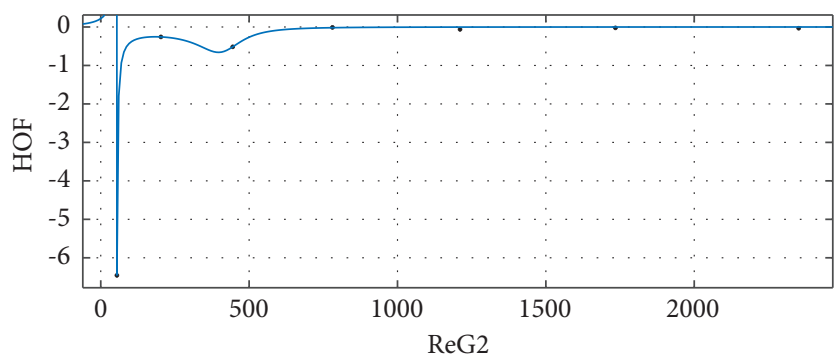

- HOF vs. ReG2

- ReG2

Figure 11: $\operatorname{HoF}\left(\operatorname{ReG}_{2}\right)$.

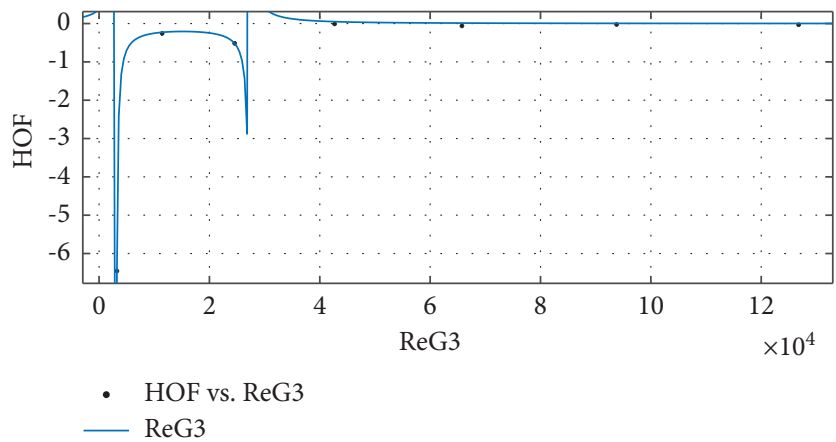

Figure 12: $\operatorname{HoF}\left(\operatorname{ReG}_{3}\right)$.

Coefficients (with 95\% confidence bounds) are given in Table 15.

5.2. General Models for Indices vs. Entropy. This section establishes a mathematical framework between each topological index and entropy of terbium oxide. All the fitted curves are shown in Figures 13-19 while the estimated parametric values are given in Tables 16-22.

$$
\begin{aligned}
\operatorname{Entropy}(\mathrm{HM}) & =\frac{p_{1} \times \mathrm{HM}+p_{2}}{(\mathrm{HM})^{3}+q_{1} \times(\mathrm{HM})^{2}+q_{2} \times \mathrm{HM}+q_{3}} . \\
\operatorname{Entropy}(F) & =\frac{p_{1} \times F+p_{2}}{F^{3}+q_{1} \times F^{2}+q_{2} \times F+q_{3}} .
\end{aligned}
$$


Table 9: HoF vs. HM.

\begin{tabular}{lcccc}
\hline & $p_{i}$ & CI & $q_{i}$ & $C I$ \\
\hline$i=1$ & 178.2 & $(-9.679 \mathrm{e}+06,9.679 \mathrm{e}+06)$ & $1.176 \mathrm{e}+04$ & $(-6.386 \mathrm{e}+08,6.386 \mathrm{e}+08)$ \\
$i=2$ & - & - & $1.94 \mathrm{e}+04$ & $(-1.053 \mathrm{e}+09,1.053 \mathrm{e}+09)$ \\
$i=3$ & - & - & 7138 & $(-3.876 \mathrm{e}+08,3.876 \mathrm{e}+08)$ \\
\hline
\end{tabular}

Table 10: HoF vs. F.

\begin{tabular}{lcccc}
\hline & $p_{i}$ & CI & $q_{i}$ & CI \\
\hline$i=1$ & 2.805 & $(-6.115 \mathrm{e}+05,6.115 \mathrm{e}+05)$ & 688.5 & $(-1.482 \mathrm{e}+08,1.482 \mathrm{e}+08)$ \\
$i=2$ & -20.61 & $(-4.445 \mathrm{e}+06,4.445 \mathrm{e}+06)$ & 1156 & $(-2.493 \mathrm{e}+08,2.493 \mathrm{e}+08)$ \\
$i=3$ & -9.782 & $(-2.11 \mathrm{e}+06,2.11 \mathrm{e}+06)$ & 444.4 & $(-9.587 \mathrm{e}+07,9.587 \mathrm{e}+07)$ \\
\hline
\end{tabular}

TABle 11: HoF vs. AZI.

\begin{tabular}{lcccc}
\hline & $p_{i}$ & CI & $q_{i}$ & CI \\
\hline$i=1$ & -0.05294 & $(-0.3091,0.2032)$ & 1.691 & $(-1.61,4.992)$ \\
$i=2$ & -0.03912 & $(-0.4201,0.3419)$ & 0.6454 & $(-4.759,6.05)$ \\
$i=3$ & -0.005199 & $(-0.08769,0.07729)$ & -0.008204 & $(-1.979,1.962)$ \\
\hline
\end{tabular}

Table 12: HoF vs. J.

\begin{tabular}{lcccc}
\hline & $p_{i}$ & CI & $q_{i}$ & CI \\
\hline$i=1$ & -190.1 & $(-1.44 \mathrm{e}+08,1.44 \mathrm{e}+08)$ & $1.333 \mathrm{e}+04$ & $(-1.009 \mathrm{e}+10,1.009 \mathrm{e}+10)$ \\
$i=2$ & -630 & $(-4.771 \mathrm{e}+08,4.771 \mathrm{e}+08)$ & $2.228 \mathrm{e}+04$ & $(-1.687 \mathrm{e}+10,1.687 \mathrm{e}+10)$ \\
$i=3$ & -236.6 & $(-1.792 \mathrm{e}+08,1.792 \mathrm{e}+08)$ & 8555 & $(-6.479 \mathrm{e}+09,6.479 \mathrm{e}+09)$ \\
\hline
\end{tabular}

TAble 13: HoF vs. $\operatorname{Re}_{1}$

\begin{tabular}{lcccc}
\hline & $p_{i}$ & CI & $q_{i}$ & CI \\
\hline$i=1$ & -175.5 & $(-2.397 \mathrm{e}+06,2.396 \mathrm{e}+06)$ & 3781 & $(-5.159 \mathrm{e}+07,5.16 \mathrm{e}+07)$ \\
$i=2$ & -112.8 & $(-1.541 \mathrm{e}+06,1.54 \mathrm{e}+06)$ & 6438 & $(-8.788 \mathrm{e}+07,8.79 \mathrm{e}+07)$ \\
$i=3$ & - & - & 2553 & $(-3.485 \mathrm{e}+07,3.486 \mathrm{e}+07)$ \\
\hline
\end{tabular}

Table 14: HoF vs. $\operatorname{ReG}_{2}$.

\begin{tabular}{lcccc}
\hline & $p_{i}$ & CI & $q_{i}$ & CI \\
\hline$i=1$ & -0.003307 & $(-0.02053,0.01391)$ & 2.406 & $(1.359,3.452)$ \\
$i=2$ & - & - & 1.881 & $(0.1678,3.595)$ \\
$i=3$ & - & - & 0.4865 & $(-0.1403,1.113)$ \\
\hline
\end{tabular}

TAble 15: HoF vs. $\mathrm{Re}_{3}$.

\begin{tabular}{|c|c|c|c|c|}
\hline & $p_{i}$ & $\mathrm{CI}$ & $q_{i}$ & $\mathrm{CI}$ \\
\hline$i=1$ & 172.1 & $(-9.52 \mathrm{e}+06,9.52 \mathrm{e}+06)$ & $1.171 \mathrm{e}+04$ & $(-6.476 e+08,6.476 e+08)$ \\
\hline$i=2$ & - & - & $1.93 e+04$ & $(-1.067 e+09,1.067 e+09)$ \\
\hline$i=3$ & - & - & 7118 & $(-3.937 e+08,3.937 e+08)$ \\
\hline
\end{tabular}




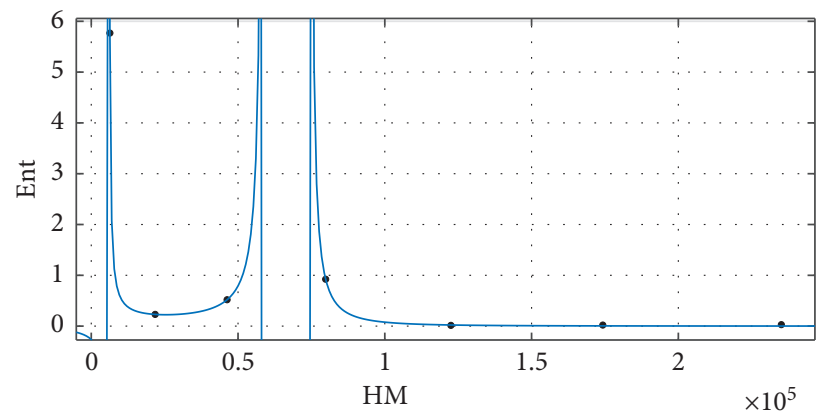

- Ent vs. HM

- HM

Figure 13: Entropy(HM).

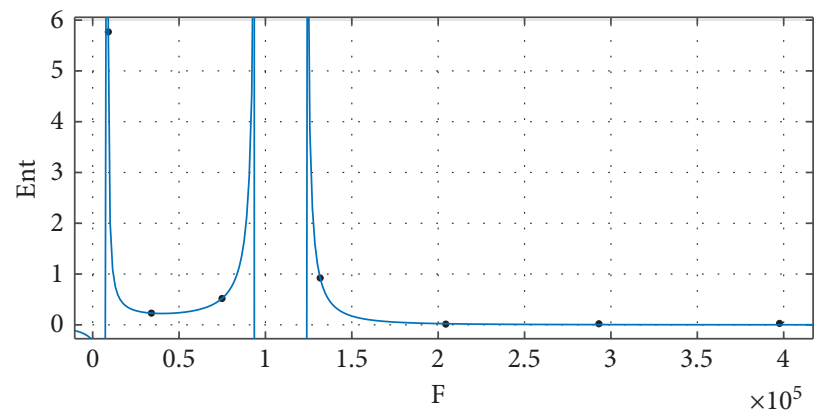

- Ent vs. F

- F

Figure 14: Entropy $(F)$.

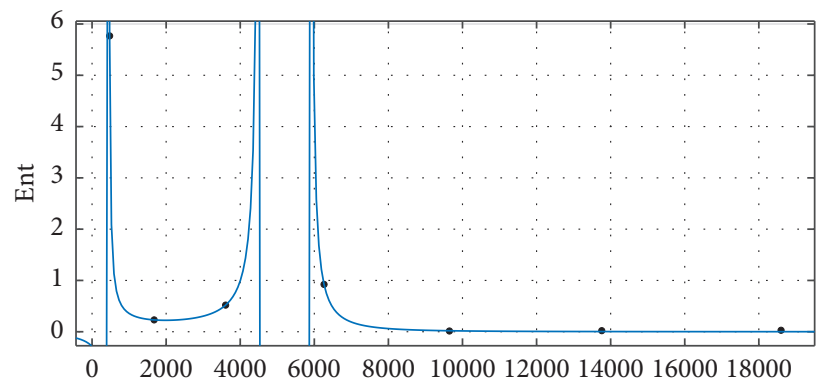

AZI

- Ent vs. AZI

— AZI

Figure 15: Entropy (AZI).

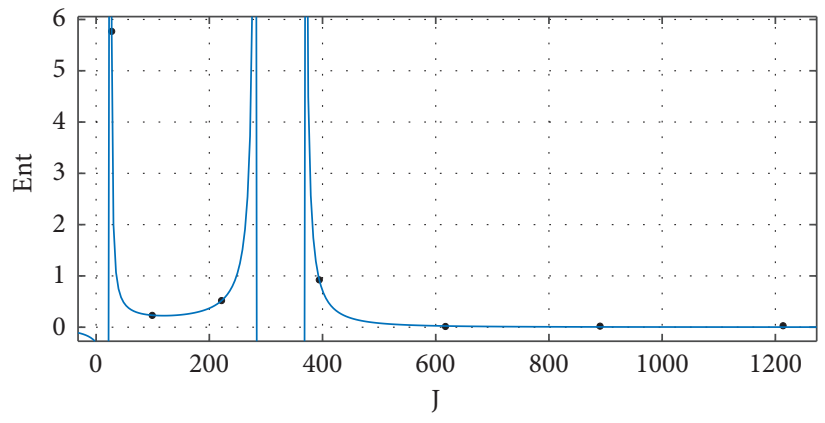

- Ent vs. J

Figure 16: Entropy $(J)$. 


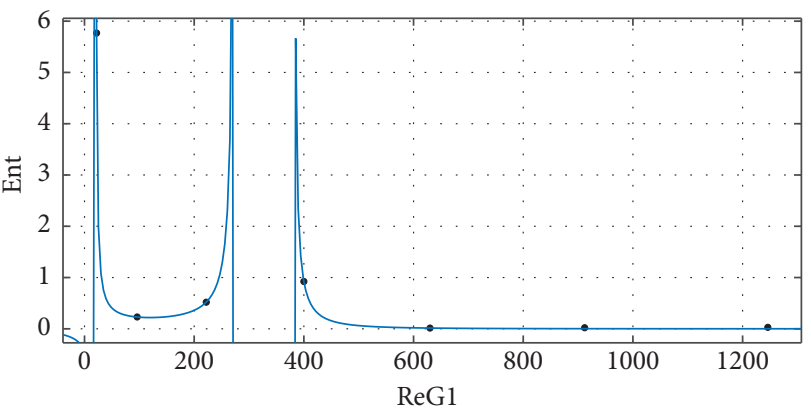

- Ent vs. ReG1

- ReG1

Figure 17: Entropy $\left(\operatorname{Re}_{1}\right)$.

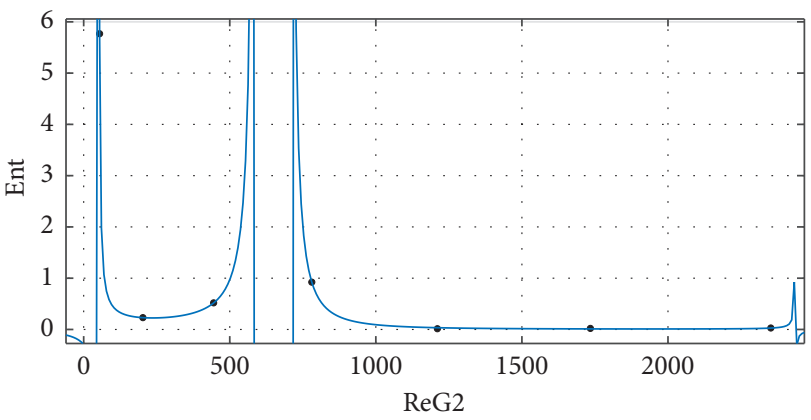

- $\quad$ Ent vs. ReG2

- ReG2

Figure 18: Entropy $\operatorname{Re}_{2}$.

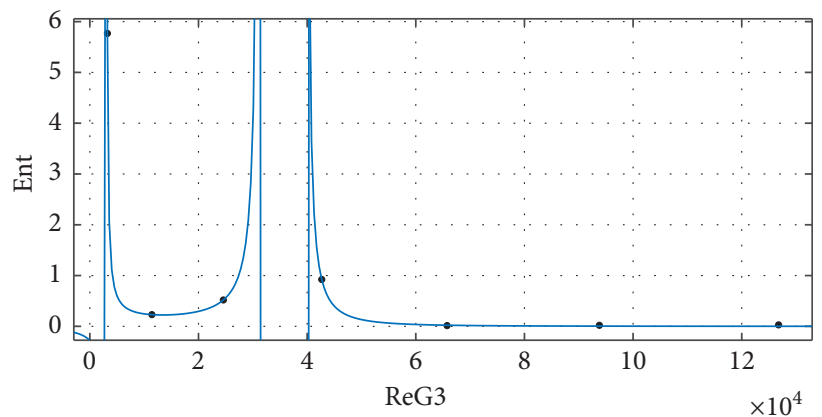

- $\quad$ Ent vs. ReG3

- ReG3

Figure 19: Entropy $\operatorname{ReG}_{3}$.

$$
\operatorname{Entropy}(\mathrm{AZI})=\frac{p_{1}}{(\mathrm{AZI})^{3}+q_{1} \times(\mathrm{AZI})^{2}+q_{2} \times \mathrm{AZI}+q_{3}},
$$

$$
\operatorname{Entropy}(J)=\frac{p_{1}}{J^{5}+q_{1} \times J^{4}+q_{2} \times J^{3}+q_{3} \times J^{2}+q_{4} \times J+q_{5}} \text {. }
$$


TABle 16: Entropy vs. HM.

\begin{tabular}{lcccc}
\hline & $p_{i}$ & CI & $q_{i}$ & CI \\
\hline$i=1$ & 0.001132 & $(-0.04439,0.04666)$ & 1.842 & $(1.762,1.922)$ \\
$i=2$ & 0.01288 & $(-0.03159,0.05736)$ & 0.9484 & $(0.8749,1.022)$ \\
$i=3$ & - & - & 0.1427 & $(0.1092,0.1761)$ \\
\hline
\end{tabular}

TABle 17: Entropy vs. $F$.

\begin{tabular}{lcccc}
\hline & $p_{i}$ & CI & $q_{i}$ & CI \\
\hline$i=1$ & 0.001791 & $(-0.04354,0.04713)$ & 1.845 & $(1.766,1.925)$ \\
$i=2$ & 0.01254 & $(-0.03159,0.05667)$ & 0.9604 & $(0.8889,1.032)$ \\
$i=3$ & - & - & 0.1465 & $(0.1139,0.1792)$ \\
\hline
\end{tabular}

Table 18: Entropy vs. AZI.

\begin{tabular}{lcccc}
\hline & $p_{i}$ & CI & $q_{i}$ & CI \\
\hline$i=1$ & 0.01144 & $(0.007304,0.01558)$ & 1.845 & $(1.812,1.877)$ \\
$i=2$ & - & - & 0.9537 & $(0.9148,0.9925)$ \\
$i=3$ & - & - & 0.1431 & $(0.1392,0.147)$ \\
\hline
\end{tabular}

TABle 19: Entropy vs. J.

\begin{tabular}{lcccc}
\hline & $p_{i}$ & CI & $q_{i}$ & CI \\
\hline$i=1$ & -110.9 & $(-2.497 \mathrm{e}+07,2.497 \mathrm{e}+07)$ & 2563 & $(-5.765 \mathrm{e}+08,5.765 \mathrm{e}+08)$ \\
$i=2$ & - & - & -3952 & $(-8.901 \mathrm{e}+08,8.901 \mathrm{e}+08)$ \\
$i=3$ & - & - & $-1.355 \mathrm{e}+04$ & $(-3.052 \mathrm{e}+09,3.052 \mathrm{e}+09)$ \\
$i=4$ & - & - & -8056 & $(-1.814 \mathrm{e}+09,1.814 \mathrm{e}+09)$ \\
$i=5$ & - & -1311 & $(-2.951 \mathrm{e}+08,2.951 \mathrm{e}+08)$ \\
\hline
\end{tabular}

TABle 20: Entropy vs. $\operatorname{Re}_{1}$.

\begin{tabular}{lcccr}
\hline & $p_{i}$ & CI & $q_{i}$ & CI \\
\hline$i=1$ & 1.041 & $(-1.206 \mathrm{e}+06,1.206 \mathrm{e}+06)$ & -1145 & $(-1.341 \mathrm{e}+09,1.341 \mathrm{e}+09)$ \\
$i=2$ & -10.05 & $(-1.175 \mathrm{e}+07,1.175 \mathrm{e}+07)$ & -2126 & $(-2.487 \mathrm{e}+09,2.487 \mathrm{e}+09)$ \\
$i=3$ & - & - & -1121 & $(-1.311 \mathrm{e}+09,1.311 \mathrm{e}+09)$ \\
$i=4$ & - & - & -170.7 & $(-1.996 \mathrm{e}+08,1.996 \mathrm{e}+08)$ \\
\hline
\end{tabular}

TABle 21: Entropy vs. $\operatorname{Re}_{2}$.

\begin{tabular}{|c|c|c|c|c|}
\hline & $p_{i}$ & $\mathrm{CI}$ & $q_{i}$ & $\mathrm{CI}$ \\
\hline$\overline{i=1}$ & 103.9 & $(-2.399 e+06,2.399 e+06)$ & -3508 & $(-8.104 e+07,8.103 e+07)$ \\
\hline$i=2$ & - & - & -384.3 & $(-8.831 e+06,8.83 e+06)$ \\
\hline$i=3$ & - & - & 7836 & $(-1.81 \mathrm{e}+08,1.81 \mathrm{e}+08)$ \\
\hline$i=4$ & - & - & 5312 & $(-1.227 \mathrm{e}+08,1.227 \mathrm{e}+08)$ \\
\hline$i=5$ & - & - & 909.8 & $(-2.101 \mathrm{e}+07,2.101 \mathrm{e}+07)$ \\
\hline
\end{tabular}

TABle 22: Entropy vs. $\operatorname{Re}_{3}$.

\begin{tabular}{lcccc}
\hline & $p_{i}$ & CI & $q_{i}$ & CI \\
\hline$i=1$ & 0.001368 & $(-0.04409,0.04682)$ & 1.843 & $(1.763,1.923)$ \\
$i=2$ & 0.01276 & $(-0.0316,0.05712)$ & 0.9527 & $(0.8799,1.025)$ \\
$i=3$ & - & - & 0.144 & $(0.1109,0.1772)$ \\
\hline
\end{tabular}




$$
\begin{aligned}
& \operatorname{Entropy}\left(\operatorname{Re} G_{1}\right)=\frac{p_{1} \times \operatorname{Re} G_{1}+p_{2}}{\left(\operatorname{Re} G_{1}\right)^{4}+q_{1} \times\left(\operatorname{Re} G_{1}\right)^{3}+q_{2} \times\left(\operatorname{Re}_{1}\right)^{2}+q_{3} \times \operatorname{Re} G_{1}+q_{4}} \text {. } \\
& \text { Entropy }\left(\operatorname{ReG} G_{2}\right)=\frac{p_{1}}{\left(\operatorname{Re} G_{2}\right)^{5}+q_{1} \times\left(\operatorname{ReG}_{2}\right)^{4}+q_{2} \times\left(\operatorname{ReG}_{2}\right)^{3}+q_{3} \times\left(\operatorname{ReG}_{2}\right)^{2}+q_{4} \times \operatorname{Re} G_{2}+q_{5}} \text {. } \\
& \operatorname{Entropy}\left(\operatorname{ReG}_{3}\right)=\frac{p_{1} \times \operatorname{Re}_{3}+p_{2}}{\left(\operatorname{Re} G_{3}\right)^{3}+q_{1} \times\left(\operatorname{Re} G_{3}\right)^{2}+q_{2} \times \operatorname{Re}_{3}+q_{3}} \text {. }
\end{aligned}
$$

Coefficients (with 95\% confidence bounds) are given in Table 16.

Coefficients (with 95\% confidence bounds) are given in Table 17.

Coefficients (with 95\% confidence bounds) are given in Table 18.

Coefficients (with 95\% confidence bounds) are given in

Table 19.

Coefficients (with 95\% confidence bounds) are given in Table 20.

Coefficients (with $95 \%$ confidence bounds) are given in Table 21.

Coefficients (with 95\% confidence bounds) are given in Table 22.

\section{Conclusions}

A connection between topological indices and thermodynamic properties of terbium IV oxide has been developed. This study helps to understand the chemical structure of terbium IV oxide based on the graphical properties of its underlying graph more deeply as this was economical and more efficient. Curve fitting techniques have been utilized to establish such a relation among indices and heat of formation and entropy. The rational fitting approach was selected based on its efficacy. This direct connection might help to explore the dynamical properties of this terbium IV oxide.

\section{Data Availability}

The data used to support the findings of this study are cited at relevant places within the text as references.

\section{Conflicts of Interest}

The authors declare no conflicts of interest.

\section{Authors' Contributions}

All authors contributed equally to this work.

\section{References}

[1] S. P. Sinha, "Systematics and the properties of the lanthanides," Springer Science and Business Media, vol. 109, pp. 33-43, 2012.

[2] V. G. Minkina, "Initial compounds for obtaining high-temperature superconducting films by the CVD-method," Russian Chemical Bulletin, vol. 42, no. 9, pp. 1460-1466, 1993.

[3] S. Abanades, "Metal oxides applied to thermochemical watersplitting for hydrogen production using concentrated solar energy," ChemEngineering, vol. 3, no. 3, pp. 63-83, 2019.

[4] S. Yang, S. Huang, M. Schnee, Q. T. Zhao, J. Schubert, and K. J. Chen, "Enhancement-mode LaLuO3-AlGaN/GaN metal-insulator-semiconductor high-electron-mobility transistors using fluorine plasma ion implantation," Japanese Journal of Applied Physics, vol. 52, no. 8S, pp. 88-98, 2013.

[5] S. Pavunny, J. Scott, and R. Katiyar, "Lanthanum gadolinium oxide: a new electronic device material for CMOS logic and memory devices," Materials, vol. 7, no. 4, pp. 2669-2696, 2014.

[6] P. R. Armstrong, M. L. Mah, S. S. Kim, and J. J. Talghader, "Thermoluminescence of Y2O3:Tb3+ thin films deposited by electron beam evaporation," Journal of Luminescence, vol. 148, pp. 225-229, 2014.

[7] S. Shu Yang, S. Sen Huang, H. Hongwei Chen, C. Chunhua Zhou, Q. Qi Zhou, M. Schnee et al., "AlGaN/GaN MISHEMTs with high- $\kappa \mathrm{LaLuO}_{3}$ gate dielectric," IEEE Electron Device Letters, vol. 33, no. 7, pp. 979-981, 2012.

[8] A. Ziani, C. Davesnne, C. Labbé et al., "Annealing effects on the photoluminescence of terbium doped zinc oxide films," Thin Solid Films, vol. 553, pp. 52-57, 2014.

[9] S. Singh, A. Singh, B. C. Yadav, and P. K. Dwivedi, "Fabrication of nanobeads structured perovskite type neodymium iron oxide film: its structural, optical, electrical and LPG sensing investigations," Sensors and Actuators B: Chemical, vol. 177, pp. 730-739, 2013.

[10] H. A. Miran, M. Altarawneh, Z. N. Jaf, B. Z. Dlugogorski, and Z. T. Jiang, "Structural, electronic and thermodynamic properties of bulk and surfaces of terbium oxide (TbO2)," Materials Research Express, vol. 5, no. 8, pp. 85-90, 2018.

[11] G. H. Shirdel, H. Rezapour, and A. M. Sayadi, "The hyperZagreb index of graph operations," Iranian Journal of Mathematical Chemistry, vol. 4, no. 2, pp. 213-220, 2013.

[12] M. Ghorbani and N. Azimi, "Note on multiple Zagreb indices," Iranian Journal of Mathematical Chemistry, vol. 3, no. 2, pp. 137-143, 2012. 
[13] M. K. Siddiqui, M. Imran, and A. Ahmad, "On Zagreb indices, Zagreb polynomials of some nanostar dendrimers," Applied Mathematics and Computation, vol. 280, pp. 132-139, 2016.

[14] M. K. Siddiqui, M. Naeem, N. A. Rahman, and M. Imran, "Computing topological indices of certain networks," Journal of Optoelectronics and Advanced Materials, vol. 18, pp. 884892, 2016.

[15] I. Gutman and N. Trinajstić, "Graph theory and molecular orbitals. Total -electron energy of alternant hydrocarbons," Chemical Physics Letters, vol. 17, no. 4, pp. 535-538, 1972.

[16] B. Furtula and I. Gutman, "A forgotten topological index," Journal of Mathematical Chemistry, vol. 53, no. 4, pp. 11841190, 2015.

[17] B. Furtula, A. Graovac, and D. Vukičević, "Augmented Zagreb index," Journal of Mathematical Chemistry, vol. 48, no. 2, pp. $370-380,2010$.

[18] A. T. Balaban, "Highly discriminating distance-based topological index," Chemical Physics Letters, vol. 89, no. 5, pp. 399-404, 1982.

[19] A. T. Balaban and L. V. Quintas, "The smallest graphs, trees, and 4-trees with degenerate topological index," Journal of Mathematical Chemistry, vol. 14, pp. 213-233, 1983.

[20] H. Yang, M. Imran, S. Akhter, Z. Iqbal, and M. K. Siddiqui, "On distance-based topological descriptors of subdivision vertex-edge join of three graphs," IEEE ACCESS, vol. 7, no. 1, pp. 143381-143391, 2019.

[21] S. M. Kang, M. K. Siddiqui, N. A. Rehman, M. Naeem, and M. H. Muhammad, "Topological properties of 2-dimensional silicon-carbons," IEEE Access, vol. 6, pp. 59362-59373, 2018.

[22] M. Imran, M. K. Siddiqui, M. Naeem, and M. A. Iqbal, "On topological properties of symmetric chemical structures," Symmetry, vol. 10, no. 5, pp. 173-183, 2018.

[23] P. S. Ranjini, V. Lokesha, and A. Usha, "Relation between phenylene and hexagonal squeeze using harmonic index," International Journal of Applied Graph Theory, vol. 1, no. 4, pp. 116-121, 2013.

[24] M. Imran, M. K. Siddiqui, A. Q. Baig, W. khalid, and H. Shaker, "Topological properties of cellular neural networks," Journal of Intelligent and Fuzzy Systems, vol. 37, no. 3, pp. 3605-3614, 2019.

[25] W. Gao, H. Wu, M. K. Siddiqui, and A. Q. Baig, "Study of biological networks using graph theory," Saudi Journal of Biological Sciences, vol. 25, no. 6, pp. 1212-1219, 2018.

[26] S. A. Nadeem, M. K. Siddiqui, and M. Naeem, "Topological descriptor of 2-dimensional silicon carbons and their applications," Open Chemistry, vol. 17, no. 1, pp. 1473-1482, 2019.

[27] J. B. Liu, C. Wang, S. Wang, and B. Wei, "Zagreb indices and multiplicative Zagreb indices of eulerian graphs," Bulletin of the Malaysian Mathematical Sciences Society, vol. 42, no. 1, pp. 67-78, 2019.

[28] Z. Shao, P. Wu, X. Zhang, D. Dimitrov, and J. B. Liu, "On the maximum $A B C$ index of graphs with prescribed size and without pendent vertices," IEEE Access, vol. 6, pp. 2760427616, 2018.

[29] Z. Raza, "The expected values of arithmetic bond connectivity and geometric indices in random phenylene chains," Heliyon, vol. 6, no. 7, pp. 44-59, 2020.

[30] B. Assaye, M. Alamneh, L. N. Mishra, and Y. Mebrat, "Dual skew Heyting almost distributive lattices," Applied Mathematics and Nonlinear Sciences, vol. 4, no. 1, pp. 151-162, 2019.

[31] D. Zhao, M. K. Siddiqui, S. Javed, L. Sherin, and F. Kausar, "Molecular topological indices-based analysis of thermodynamic properties of graphitic carbon nitride," The European Physical Journal Plus, vol. 135, no. 12, pp. 1-19, 2020. 